\title{
Impacto de un Programa de Renovación Metodológica en las Estrategias Cognitivas y el Rendimiento Académico en Cursos de Física Universitaria
}

\author{
Javier A. Pulgar e Iván R. Sánchez \\ Departamento de Física, Facultad de Ciencias, Universidad del Bío Bío, Casilla 5-C, Concepción, Chile. \\ (e-mail: jpulgar@ubiobio.cl)
}

Recibido May. 28, 2014; Aceptado Jul. 14, 2014; Versión final recibida Ago. 6, 2014

\begin{abstract}
Resumen
El presente trabajo, tiene por finalidad compartir las implicaciones didácticas que surgen al implementar un programa de renovación metodológica, que considera el uso de mapas conceptuales y diagramas $\mathrm{V}$ de Gowin como medio para abordar los contenidos de un curso de física general para alumnos de primer año de ingeniería civil de la Universidad del Bío-Bío en Concepción, Chile. Se analiza el impacto del programa de intervención en las estrategias de aprendizaje, intereses y realizaciones creativas y en el rendimiento académico. La investigación es de carácter cuasi-experimental con tres grupos independientes, con pre y post pruebas. El grupo experimental fue intervenido con metodologías activas y elementos heurísticos (V de Gowin y mapa conceptual), mientras que los grupos de control trabajaron resolviendo problemas a través de guías e instrucción por alumnos-ayudantes. Los resultados indican que el grupo experimental obtiene mejores resultados en el rendimiento académico y desarrolla la estrategia de procesamiento profundo.
\end{abstract}

Palabras clave: física universitaria, estrategias cognitivas, mapas conceptuales, diagramas $V$ de Gowin, metodologías activas

\section{The Impact of a Methodological Renewal in the Cognitive Strategies and Academic Performance in Courses of University Physics}

\begin{abstract}
The present work aims to share the educational implications that arise when implementing a program of methodological renewal, which considers the use of conceptual maps and Gowin's V diagrams to teach and learn the contents of a general physics course for freshmen of the civil engineering major at the University of Bío Bío in Concepción, Chile. The impact of the intervention program on learning strategies, interests and creative achievement and academic performance are analyzed. This research is quasi experimental with three independents groups and pre and post tests. The experimental group was involved with active methodologies and heuristics (Gowin's $\mathrm{V}$ and concept map), while the control groups worked through problem solving guides and teaching assistants. The results indicate that the experimental group obtained better results in academic performance and developed deep processing strategy
\end{abstract}




\section{INTRODUCCIÓN}

Existen una serie de argumentos que han motivado cambios en la forma de enseñar y aprender física en la Universidad del Bío-Bío (UBB), Concepción, Chile. El aumento en la matrícula ha conducido a un grupo heterogéneo de alumnos en las aulas, lo que implica la activación de diferentes estrategias de aprendizaje para una misma actividad académica. Respecto a esto, es posible diferenciar a los alumnos de acuerdo al tipo de estrategia que despliegan en sus respectivas áreas de estudio. Por un lado, existen alumnos que utilizan las estrategias adecuadas - según Ronald Schmeck (Cano y Justicia, 1998. pp. 99)- a las exigencias académicas - procesamiento profundo y elaborativo - y logran tener éxito (Martín et al, 2008), mientras que en la otra vereda se encuentran los que no manejan dichas herramientas y emplean otro tipo de mecanismos - retención de hechos y estudio metódico - y entonces, no logran resultados favorables durante su carrera universitaria (Fernández, Martínez y Melipillán, 2009). Las características del primer grupo se vinculan al aprovechamiento de las claves semánticas de la nueva información al momento de codificar, dar significado y construir conceptos, siendo conscientes de sus propias formas de aprender. El otro segmento, pone la atención en las características superficiales del contenido, manteniéndose pasivo frente al desarrollo de significados y en consecuencia, logran un bajo rendimiento académico. En el contexto de la Universidad del Bío-Bío, los alumnos ingresan sin haber desarrollado las estrategias pertinentes que facilitaría un aprendizaje significativo (Sánchez, 2012).

Los buenos resultados académicos, producto de un aprendizaje significativo, se encuentran relacionados con la transferencia, la que es entendida como la habilidad para adecuar el material aprendido a situaciones problemáticas en diferentes contextos, y que además, se encuentra estrechamente vinculada a las características creativas que manifiestan los sujetos. Desarrollar esta capacidad para transferir, y a su vez estimular la creatividad de los alumnos, es posible gracias a la implementación de metodologías activas que permitan enfrentar al alumno a la contextualización del conocimiento, y en virtud del número y riqueza de las situaciones problemáticas a resolver, elaborar y enriquecer su capacidad para transferir (vega et al, 2014). Por otro lado, un aprendizaje con significado se constituye en la medida en que la nueva información sea reflexionada y organizada en la estructura cognitiva del sujeto que se dispone a aprender. De esta forma, instrumentos como el diagrama $V$ de Gowin y el mapa conceptual, resultan de gran ayuda para la manifestación del cómo se entiende la información y qué significados se le otorga (Pulgar y Sánchez, 2013). Mientras la V de Gowin devela los vínculos entre las teorías y los procedimientos, el mapa permite graficar el dominio conceptual del alumno, favoreciendo la integración del contenido. En ello radica la importancia que adquieren estos elementos heurísticos en el proceso de enseñar-aprender (Moreira 2006).

En relación a lo anterior, se elaboró un programa de intervención para la enseñanza y aprendizaje de Cinemática y Dinámica, utilizando metodologías activas como aprendizaje basado en problemas (ABP), guías de preguntas creativas, junto a mapas conceptuales y diagramas $V$ de Gowin. El objetivo es desarrollar las estrategias cognitivas (aprendizaje y creativas) adecuadas para que los alumnos logren construir significados en relación al conocimiento en física y así obtener un alto rendimiento académico. El programa opera introduciendo ABP y preguntas creativas previa formalización de los contenidos como actividades de búsqueda y selección de la información, con el objetivo de generar el conflicto cognitivo y motivar la aproximación al nuevo contenido. Luego, el mapa de conceptos se integra como actividad para potenciar la reflexión e identificar el dominio conceptual y los significados construidos por los estudiantes. Finalmente se hace uso de la $\mathrm{V}$ de Gowin para resolver problemas integrando el dominio teórico y procedimental (Pulgar, Sánchez, 2013).

Los resultados indican, que los alumnos sometidos a la intervención metodológica obtienen mejores resultados en rendimiento académico y logran elevar los puntajes en la estrategia de procesamiento profundo, lo que resulta fundamental en la transición del aprendizaje mecánico hacia el aprendizaje con significado. Por otro lado, se deja entrever la relación entre el rendimiento académico, niveles de creatividad y tipos de aprendizaje, este último definido en función de las estrategias de aprendizaje que se activan frente a una tarea. En consecuencia, es posible declarar que los tipos de aprendizaje estratégico y significativo pronostican el éxito académico, así como los altos niveles de creatividad.

\section{FUNDAMENTOS TEÓRICOS}

\section{Estrategias de aprendizaje y aprendizaje significativo}

Cuando un estudiante se sumerge en un ambiente de aprendizaje, él responde, percibe y reacciona desplegando una serie de mecanismos conocidos como estrategias de aprendizaje. Estas pueden ser entendidas como el conjunto consciente, intencionado y organizado de aquello que debe hacer el alumno para lograr el éxito frente a una tarea. Un autor logra definirlas como "un conjunto de acciones que se realizan para obtener un objetivo de aprendizaje" (Martínez, 2009).. Sin embargo, hoy logran ser definidas 
como mecanismos de toma de decisiones, conscientes e intencionales, que implican la selección de los conocimientos conceptuales, procedimentales y actitudinales, pertinentes para lograr un objetivo académico, en una determinada situación educativa (Gargallo, 2007). En consecuencia, las estrategias de aprendizaje se constituyen en un elemento fundamental en el ejercicio de aprender a aprender, guardando una estrecha relación con el rendimiento académico (Gargallo, 2006; Martín et al, 2008; Carbonero et al, 2010).

En el modelo de Schmeck (1988) se definen cuatro estrategias de aprendizaje: procesamiento profundo, procesamiento elaborativo, estudio metódico y retención de hecho, Truffello y Pérez (1998), de tal forma que el éxito en el aprendizaje reside en que la información se procese profunda y elaborativamente (Cid, 2008; Fernández, Martínez y Melipillán, 2009). En consecuencia, una estrategia de aprendizaje "adecuada", en el contexto de la educación formal universitaria, será aquella orientada hacia un procesamiento profundo y elaborativo. Utilizando esto es posible definir tres tipos de aprendizaje (significativo, estratégico y mecánico), en virtud de las estrategias cognitivas por las que muestran preferencia los alumnos. El aprendizaje significativo está marcado por la orientación hacia el procesamiento profundo y elaborativo de la información, mientras que un aprendizaje estratégico se caracteriza por el procesamiento profundo o elaborativo, sumado al estudio metódico. $Y$ entonces, un aprendizaje mecánico tendrá como estrategias fundamentales el estudio metódico y la retención de hecho.

\section{Creatividad y aprendizaje significativo}

Es posible señalar que la creatividad es un mecanismo inherente a la mente humana y sensible a los problemas, que se activa para crear soluciones (o problemas) originales, diversos y flexibles, que buscan la transformación de la realidad presente de individuo. Por su lado, Ausubel et al (1983) le otorga a la creatividad una visión cognitiva, señalando que esta comienza con simples reconciliaciones integradoras que guían la construcción de nuevos conceptos, jerarquizando la estructura cognitiva del sujeto y favoreciendo la aparición de relaciones creativas.

La clave que permite develar la unión entre la creatividad y el aprendizaje significativo, emerge de la definición de la primera y se constituye en uno de sus objetivos: transformar. Bien es sabido también, que las evidencias de un aprendizaje con significado radican en que el sujeto sea capaz de adecuar (transformar) lo aprendido a situaciones contextuales diversas y llegar a soluciones sin dificultades, entendida esta como su habilidad de transferencia. En la misma línea, Donolo y Rinaudo (2008) señalan que "el proceso de transferir y utilizar conocimientos en situaciones nuevas requiere de una actitud creativa, que permitiría a los alumnos dar un salto, ante tareas poco conocidas y algo desestructuradas, hacia nuevas aplicaciones de conceptos en contextos diferentes al de aprendizaje." En la misma línea, Chroback (2008) indica que la actividad creativa requiere de conocimiento conceptualmente organizado y de motivación para crear, siendo ambos elementos favorecidos por un aprendizaje significativo. Carpio et al (2007), señala que la conducta creativa no puede ser enseñada, pero si promovida a través del enriquecimiento tanto en la forma de resolver, como en el tipo de problemas que son planteados dentro de un área de estudios. De esta forma establece un continuo entre la conducta inteligente y la conducta creativa, lo que implica que el sujeto puede ser creativo siempre y cuando, aquello que va a ser sometido a transformación se encuentre presente en su estructura cognitiva, es decir, se haya aprendido previamente.

\section{Aprendizaje basado en problemas y preguntas creativas}

El Aprendizaje Basado en Problemas, (desde ahora ABP) viene siendo utilizado en la formación universitaria desde los años 60 del siglo pasado, cuando fue planteado como alternativa a la formación tradicional en la Universidad de McCaster (Canadá). Desde entonces se ha transformado en una poderosa herramienta para la enseñanza de las disciplinas científicas, permitiendo abordar los contenidos desde la perspectiva de acontecimientos atractivos y estimulantes (Vega, et al; 2014). En el contexto de la física, el sin número de situaciones existentes y relacionables con las materias a enseñar, enriquece la capacidad de darle significado a los conceptos (Sánchez, 2012). Por esta razón, los países más aventajados desde el punto de vista educativo, lo utilizan en todos los niveles, generando mejoras en la motivación, lo que conduce a superar los objetivos de aprendizaje, y favorece además, la elaboración de las competencias exigidas en el mundo laboral como el trabajo en equipo y el auto-aprendizaje.

Las guías de preguntas buscan favorecer el desarrollo de respuestas originales y creativas, y en consecuencia, la transferencia. Existen ciertos autores que defienden la tesis de una praxis educativa fundamentada en el desarrollo de la creatividad (Torre, 1993; Reyes, 2003; López, 2008), argumentando que una actitud creativa permite enfrentarse a lo nuevo, improvisar y dar respuesta gracias a la transferencia de lo aprendido. Por esta razón, la utilización de preguntas creativas en el proceso de enseñanza y aprendizaje permitirá enriquecer la contextualización en las tareas de estudio, y así, facilitar la transferencia. En esta misma línea se ha señalado el respeto hacia las preguntas poco comunes, como un principio a considerar por los profesores para recompensar el pensamiento creativo (Ausubel et al., 1983). 


\section{Mapas conceptuales y diagramas $V$ en la enseñanza de las ciencias}

Una imagen puede ser entendida como un elemento representacional del saber del sujeto, respecto a algún determinado tópico. Por esta razón, la educación formal ha tenido que hacer uso de estas representaciones con el fin de comprender la construcción de significados e identificar la naturaleza de las relaciones conceptuales. Es por ello que el diagrama V de Gowin y el mapa conceptual adquieren tanta importancia dentro del proceso de enseñar y aprender. Por un lado, la $\vee$ permite develar los aspectos epistemológicos en la producción del conocimiento (Moreira, 2006), ahí su importancia en la resolución de problemas, mientras que el mapa de conceptos grafica el nivel de integración conceptual en función del entramado de relaciones, constituyéndose en una poderosa herramienta para la reflexión de la información (Pulgar y Sánchez, 2013). Ambas herramientas emergen en el universo educativo para favorecer el aprendizaje significativo (Moreira, 1998; 2007), por esta razón han sido utilizadas en numerosas investigaciones educativas con el objetivo de resolver y analizar problemas o evaluar trabajos prácticos y procedimientos (Gil y Solano, 2011; Flores, Caballero y Moreira, 2009; López, Ángela y Solano 2011), donde se han logrado mejoras en la comprensión e integración de los conceptos, facilitando la construcción del conocimiento, incrementando incluso la motivación hacia la resolución de problemas. Sin embargo, como señala Perales (2008), la utilización de estas imágenes -representaciones- per se, no logra mejorar el aprendizaje. Al respecto, el mismo autor indica que es necesario interactuar con estas representaciones, es decir, someterlas a análisis, crítica, etc., pues no deben ser consideradas como elementos de mera contemplación (Perales, 2008).

\section{METODOLOGÍA DE LA INVESTIGACIÓN}

Esta investigación se lleva a cabo a través de un diseño cuasi-experimental de tres grupos independientes (Cohen y Manion, 1990), Un Grupo experimental (GE) y dos controles $\left(G_{1}\right)$ y $\left(G_{2}\right)$, con pre y post-test en cada una de las variables de estudio: estrategias de aprendizaje; niveles de creatividad; y conocimiento en física. Además se aplica un instrumento para determinar el rendimiento académico. Cabe señalar, que los grupos cursan la asignatura Física I, en la modalidad estructura modular, donde la asignatura se divide en dos módulos (I y II) que agrupan los contenidos de Cinemática y Dinámica (Módulo I), y Colisiones y Rototraslación (Módulo II). La Tabla 1 representa el diseño de investigación. En la Tabla, O $\mathrm{O}_{1}$ y O' es Inventario de Estrategias de Aprendizaje de Ronald Schmeck; $\mathrm{O}_{2}$ y $\mathrm{O}_{2}$ es el Cuestionario de Intereses y Realizaciones Creativas (CIRC); $\mathrm{O}_{3}$ es el Certamen de Física; $\mathrm{T}_{1}$ es el Programa basado en metodologías activas, mapas y diagramas $\mathrm{V} ; \mathrm{T}_{2}$ es el Programa basado en resolución de guías de trabajo; y $\mathrm{T}_{3}$ es el Programa basado en instrucción entre pares.

Tabla 1: Representación del diseño.

\begin{tabular}{|c|c|c|c|}
\hline Grupos & Pre-test & Tratamiento & Post-test \\
\hline$G E$ & $\mathrm{O}_{1} \mathrm{y}_{2}$ & $\mathrm{~T}_{1}$ & $\mathrm{O}_{1}^{\prime}, \mathrm{O}_{2}^{\prime} \mathrm{y} \mathrm{O}_{3}^{\prime}$ \\
\hline $\mathrm{GC}_{1}$ & $\mathrm{O}_{1} \mathrm{y}_{2}$ & $\mathrm{~T}_{2}$ & $\mathrm{O}_{1}^{\prime}, \mathrm{O}_{2}^{\prime} \mathrm{y} \mathrm{O}_{3}$ \\
\hline $\mathrm{GC}_{2}$ & $\mathrm{O}_{1} \mathrm{y}_{2}$ & $\mathrm{~T}_{3}$ & $\mathrm{O}_{1}^{\prime}, \mathrm{O}_{2}^{\prime} \mathrm{y} \mathrm{O}_{3}$ \\
\hline
\end{tabular}

\section{Población y muestra}

La población corresponde a todos los alumnos de primer año de Ingeniería Civil en la Universidad del BíoBío. La muestra la componen los alumnos de primer año que participan de la asignatura Física I de carácter modular. En consecuencia, la muestra corresponde a 113 sujetos distribuidos en los tres grupos.

\section{Instrumento para la recogida de datos}

En esta investigación se utilizaron tres instrumentos para la acumulación de datos. Las características de cada uno de ellos y el objetivo que se cumple en el estudio, se indican a continuación:

A) Cuestionario de Intereses y Realizaciones Creativas (CIRC): test que permite identificar las capacidades creativas de los alumnos. Cuenta con 60 ítems agrupados en cinco criterios: 1. Creatividad en Arte y Escritura (AE), 2. Desafío e Inventiva (DI), 3. Confianza e Independencia (Cl), 4. Imaginación (I) e 5. Intereses Variados (IV). Los valores obtenidos a través del test Alpha de Cronbach, para la validez del instrumento son: Creatividad en Arte y Escritura: $\alpha=0,832$; Desafío e Inventiva: $\alpha=0,713$; Confianza e Independencia: $\alpha=0,694$; Imaginación: $\alpha=0,735$; Intereses Variados: $\alpha=0,70$. Estimación de la confiabilidad para la prueba total: $\alpha=0,90$. 
B) Inventario de estrategias de aprendizaje de Ronald Schmeck, adaptado para Chile (Truffello y Pérez, 1998), que cuenta de 55 enunciados distribuidos en cuatro dimensiones: Procesamiento Profundo (PP), Procesamiento Elaborativo (PE), Estudio Metódico (EM) y Retención de Hecho (RH)., los valores de la confiabilidad del test Alpha de Cronbach se determinaron el 2008,. Los valores específicos fueron: inventario de estrategias de aprendizaje, $\alpha=0.89$; PP, $\alpha=0.93 ; \mathrm{RH}, \alpha=0.89$ (Sánchez, Neriz, y Ramis, 2008).

C) Evaluación de la asignatura, cuyo objetivo es medir el rendimiento académico. Se realiza a través de certámenes y test que son construidos por un grupo de 6 docentes, lo que asegura su validez de contenidos. Estos documentos tienen por finalidad evaluar los siguientes contenidos: cinemática en una y dos dimensiones; y dinámica de Newton.

\section{Análisis de datos}

El análisis de los datos se llevará a cabo a través de estadística no paramétrica, utilizando análisis descriptivo y univariado por medio de gráficos, además del estudio de correspondencia múltiple entre las variables involucradas. El estudio de los resultados de pre y post test se realizó a través de la prueba de Kruskal-Wallis, con el objetivo de identificar las diferencias significativas entre las puntuaciones de los grupos. Frente a la presencia de diferencias estadísticamente significativas, el test de Tukey permitirá declarar a favor de qué grupo se generan los cambios. Para probar la efectividad de la metodología en un mismo grupo, se aplicó la prueba de Mc-Nemar.

\section{Propuesta de Aula}

A continuación, se ilustra la forma de trabajar en el aula a través de dos métodos a) resolución de problemas usando la $\mathrm{V}$ de Gowin y b) El método de las preguntas, ambos utilizados para abordar los contenidos del módulo, La otra metodología utilizada Aprendizaje basado en problemas se puede revisar en Sánchez, et al., (2009 y 2011).

Resolución de problemas a través de $\mathrm{V}$ de Gowin: En la figura 1 se ilustra este método, se observa como los principios y conceptos (Dominio Teórico) interactúan con las Transformaciones y Registros (Dominio Metodológico) con el fin de responder la pregunta (Foco) y elaborar Aseveraciones de Conocimiento a modo de respuesta.

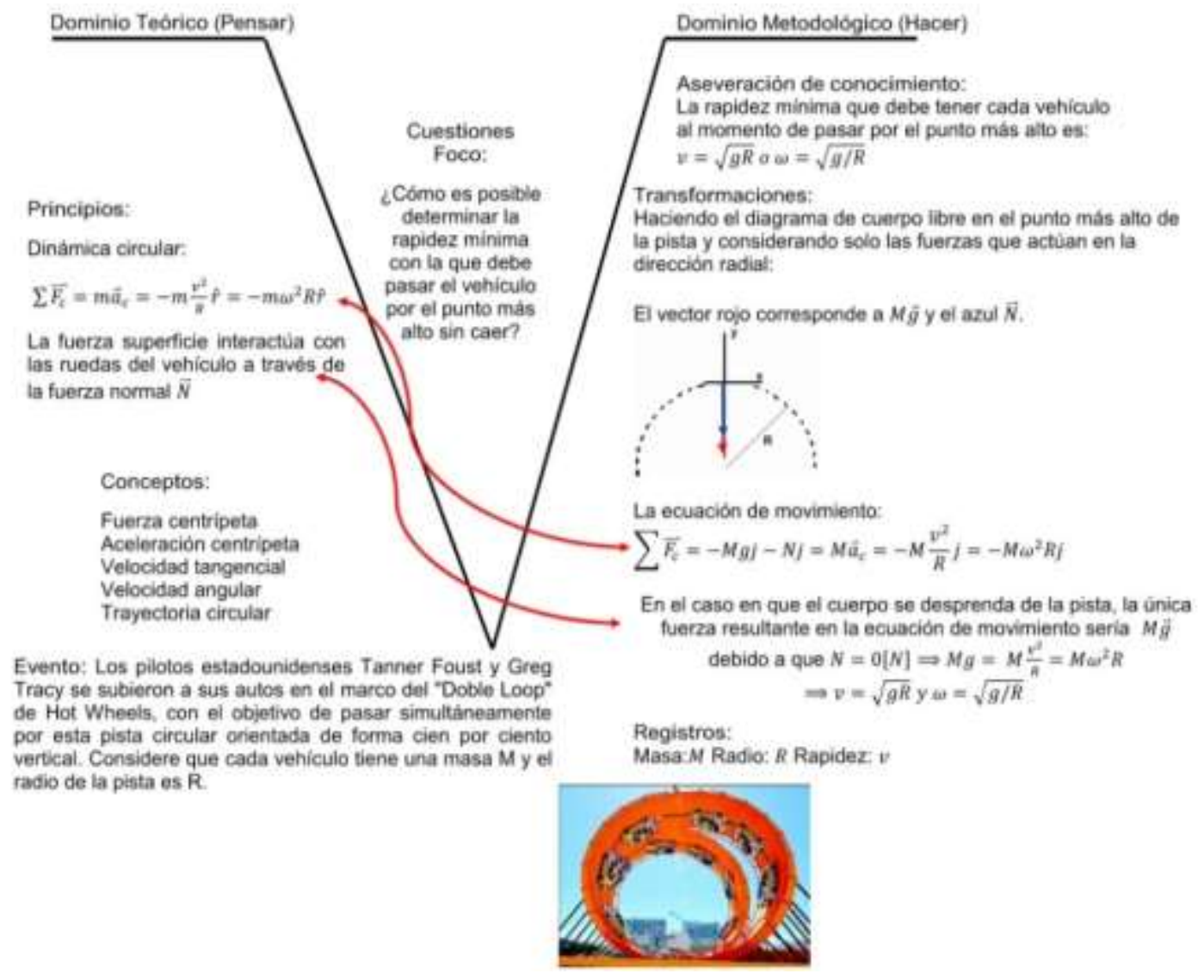

Fig. 1: Ejemplo de problema resuelto mediante el diagrama $V$ de Gowin. 
Guía de preguntas para el aprendizaje significativo: Para ilustrar algunas características del método de preguntas creativas, se considera el siguiente escenario. Considere la situación de la Figura. V, en la que un cañón lanza una bala desde una altura $\mathrm{h}$ con un ángulo de inclinación $\theta$ y una rapidez inicial Vo. Con su grupo de trabajo asigne valores de rapidez inicial Vo, altura $\mathrm{h}$ y ángulo de inclinación $\theta$ en la figura y respondan a las siguientes preguntas planteadas.

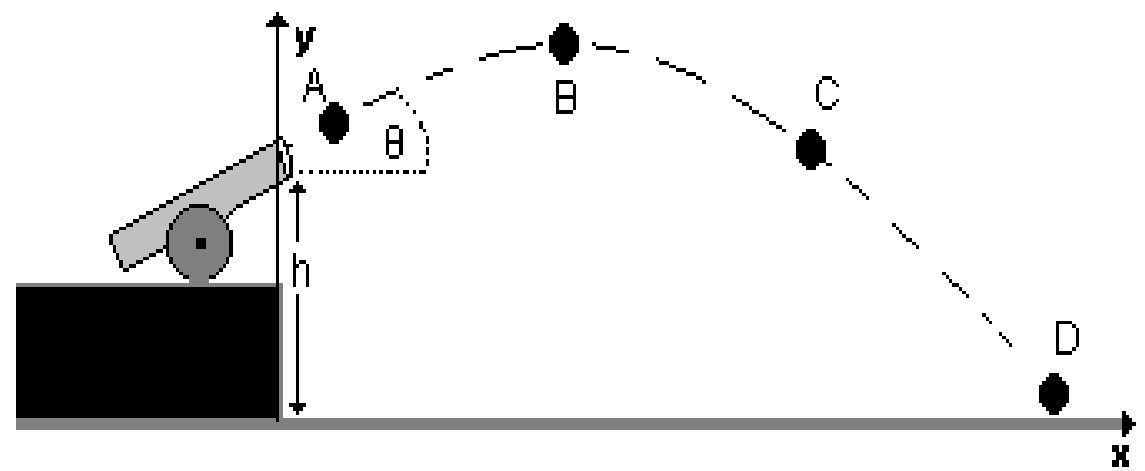

Fig. 2: Movimiento de una bala o proyectil.

a) Preguntas fácticas: ¿Con este sistema de referencia, la velocidad de la bala es siempre positiva?; ¿Qué tipo de movimiento lleva la bala al pasar por el punto $A$ y al pasar por $C$ ?

b) Preguntas de comprensión: Encuentre las ecuaciones (integrando) que describen el movimiento de la bala; ¿Cuál es la posición y velocidad de la bala en el punto D?

c) Preguntas creativas: ¿Qué pasaría si la bala fuera disparada en la Luna?, ¿Qué pasaría si la aceleración de la gravedad desapareciera cuando la bala está en el punto de máxima altura?

Estos tipos de preguntas se subdividen en 5 categorías:

i) Preguntas de Interpretación: Compare el valor de la posición de la bala para los instantes B y C, De acuerdo con la Figura, ¿la rapidez es mayor en el punto A, B, C o D?;

ii) Preguntas de Aplicación: ¿En qué instante de tiempo la bala se encuentra en el punto más alto?, ¿Qué velocidad llevará la bala en el punto C que se encuentra a 10[m] del suelo?;

iii) Preguntas de Análisis: ¿Por qué razón los misiles no se ven afectados por la aceleración de la gravedad?, ¿Por qué los aviones no describen una trayectoria parabólica?;

iv) Preguntas de Síntesis: Desarrolle un mapa conceptual con los conceptos involucrados en este problema, Realice un diagrama con las etapas de la solución del problema; y

v) Preguntas de Evaluación; ¿Qué pasaría si la aceleración de gravedad se triplicase al disparar la bala?, ¿Qué pasaría si la bala se dispara en la Luna?

\section{RESULTADOS}

Pre y post test CIRC, comparación entre tres grupos independiente

Los resultados promedios obtenidos del pre y post-test de intereses y realizaciones creativas se muestran en la figura 3 y 4 . La primera medición se realiza para determinar la homogeneidad de la muestra antes de la intervención y la segunda medición para establecer la influencia del programa en los niveles de creatividad. En la figura 3 se observa que entre los grupos no existen grandes diferencias en los puntajes obtenidos en cada una de las categorías antes de comenzar la asignatura. Esto se corrobora mediante la prueba estadística de Kruskal-Wallis, que permite determinar que no existen diferencias significativas a favor de ninguna sección. De tal forma, es posible señalar que la muestra es homogénea en relación a los niveles de creatividad, en consecuencia, se establece que las diferencias en las medidas del post-test, pueden ser producto de la metodología utilizada. De la figura 4 se observa una diferenciación de los puntajes obtenidos por el grupo experimental, en las categorías Desafío e Inventiva (DI), Confianza e Independencia $(\mathrm{Cl})$, e Intereses Variados (IV). Sin embargo, al comparar los puntajes a través de la prueba 
estadística de Kruskal-Wallis, se determina que no existen diferencias significativas al 95\%, a favor de ningún grupo.

Resultados de Pre y post test CIRC en un mismo grupo

Para observar el impacto de cada metodología dentro de cada grupo, se elaboró un gráfico (figura 5) con el porcentaje de estudiantes que en pre y post-test obtienen puntajes dentro de la categoría normal-alto en el test CIRC. Dicho gráfico muestra - de izquierda a derecha - la evolución de la creatividad en el G. Experimental, G. Control 1 y G. Control 2.

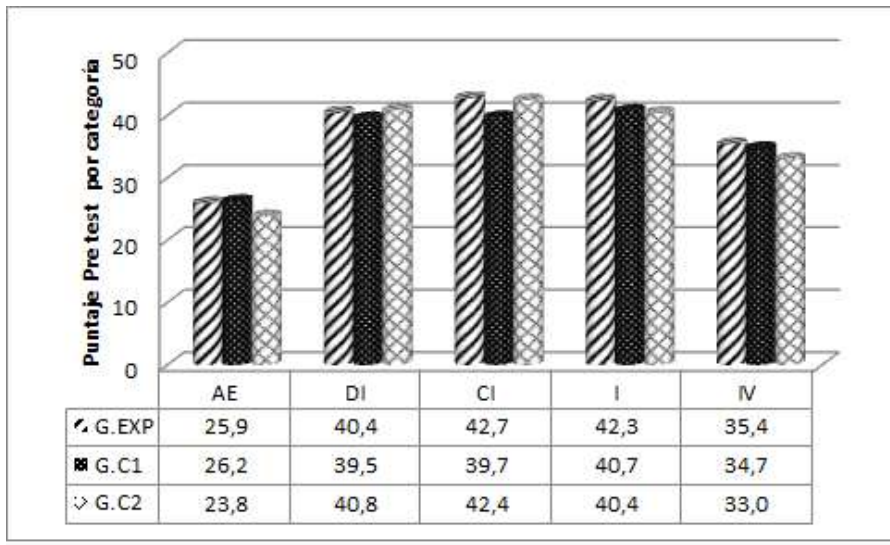

Fig. 3: Puntaje pre-test por categoría de intereses y realizaciones

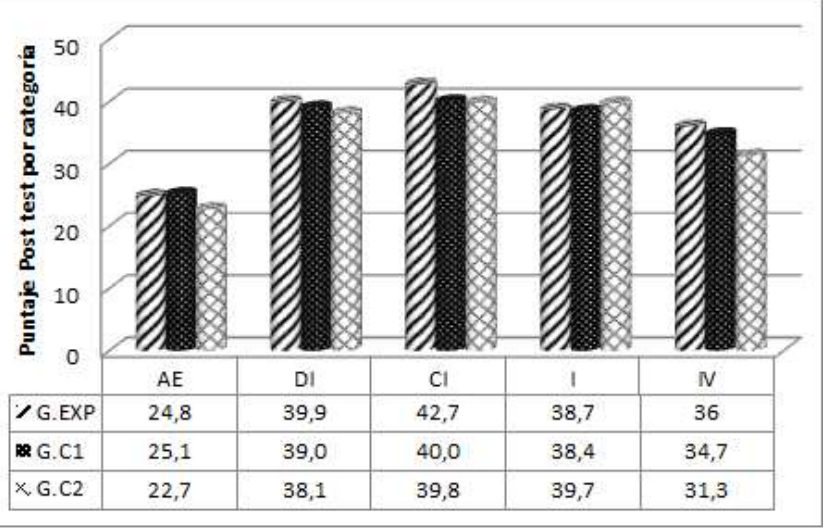

Fig. 4: Puntaje post-test por categoría de intereses y realizaciones

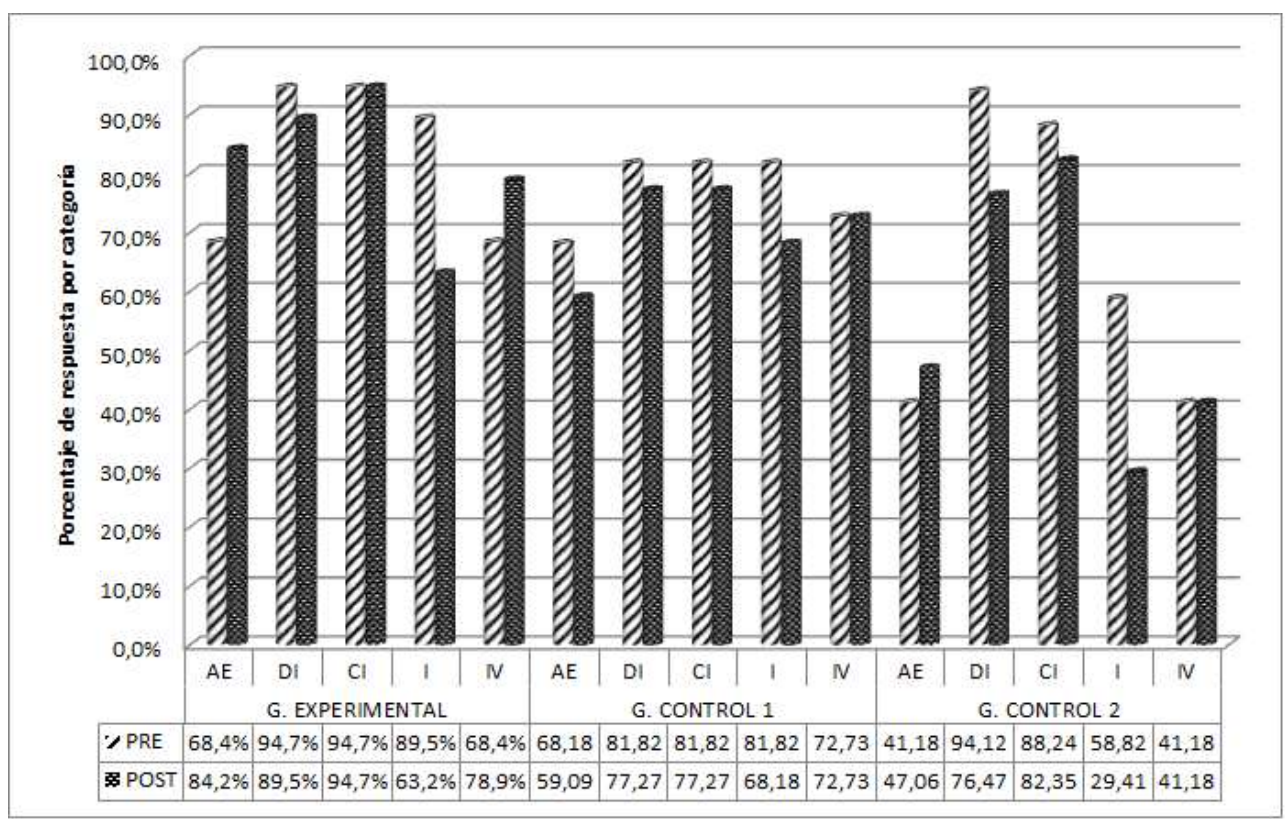

Fig. 5: Porcentaje de alumnos en nivel normal- alto para pre y post test versus categorías de creatividad. En orden: G. Experimental - G. Control $1-$ G. Control 2.

Para el Grupo Experimental es posible identificar una notoria mejoría en el porcentaje de alumnos que obtiene puntuaciones entre los rangos normal y alto, en las dimensiones Arte y Escritura (AE) e Intereses Variados (IV). A pesar de las diferencias, el análisis de los resultados mediante la prueba de McNemar sobre el grupo experimental no revela que existan diferencias significativas al $95 \%(0,05)$. Con respecto al Grupo Control 1, este mantienen los puntajes en Intereses Variados (IV), mientras que el porcentaje de alumnos con resultados normal-alto decrece en cada una de las otras categorías, salvo en Confianza e Independencia $(\mathrm{Cl})$. Mediante la prueba de McNemar, se comprueba que ninguno de estos cambios es significativo. El grupo control 2 manifiesta mejoras sólo en Arte y Escritura (AE), bajando la puntuación en el resto de las categorías. A pesar de los cambios, se demuestra que estos no son significativos en un $95 \%$. 


\section{RESULTADOS INVENTARIO DE ESTRATEGIAS DE APRENDIZAJE}

\section{Pre y post-test inventario de estrategias de aprendizaje comparación de tres grupos independientes}

Con el fin de identificar la homogeneidad de la muestra y el impacto de la intervención se aplica el cuestionario de estrategias de aprendizaje de R. Schmeck antes y después de comenzar la intervención para establecer el impacto de la propuesta metodológica. Las figuras 6 y 7 muestran los puntajes promedio obtenidos por cada grupo en el pre y post-test. A través de esto, se hace posible comparar las mediciones antes de la intervención para estudiar la homogeneidad de la muestra y después de la intervención para establecer la influencia de la intervención, en las diferentes categorías de las estrategias de aprendizaje, que permite obtener la orientación hacia el aprendizaje significativo, estratégico o mecánico. De la figura 6 , se deduce que no existen grandes diferencias en cada una de las categorías del cuestionario de estrategias de aprendizaje entre los grupos, lo que se corrobora a través de la prueba estadística de Kruskal-Wallis, que muestra que no existen diferencias estadísticamente significativas entre los grupos. Es decir, la muestra es homogénea en relación a las estrategias de aprendizaje, de tal forma, que cualquier evidencia de cambio en algunas de sus categorías tiene un alto \% de ser producto de intervención en el aula.

De la figura 7 muestra que el grupo experimental logra mejoras en la categoría Procesamiento Profundo (PP), además de reducir los puntajes obtenidos en las categorías Estudio Metódico (EM) y Retención de Hecho $(\mathrm{RH})$, estas últimas son características de un aprendizaje mecánico, lo que indicaría una transición del aprendizaje mecánico, hacia el estratégico o significativo, que exigen transferencia de contenido lo que se evidencia en un aumento del procesamiento profundo y elaborativo. Por otro lado, los grupos control $1 \mathrm{y}$ 2, sólo logran incrementar sus puntajes en la estrategia de Retención de Hecho $(\mathrm{RH})$, lo que indicaría una conducción hacia un aprendizaje superficial y reiterativo. Mediante la prueba estadística de Kruskal-Wallis, se establece un estadístico $\mathrm{H}=6,677369$ y un nivel de significado $\mathrm{p}=0,0355(96,6 \%)$ que permite afirmar que existen diferencias estadísticamente significativas en la estrategia de Procesamiento Profundo (PP) entre los grupos. Al aplicar el test de Tukey se establecen diferencias estadísticamente significativas a favor del Grupo Experimental respecto al grupo Control 1; $p=0,028321(97, \%)$ pero respecto al Grupo Control 2; $p=$ $0,7736996(92 \%)$; también se observa que entre los grupos Controles no hay diferencias significativas $\mathrm{p}=0,179212(82 \%)$, estas diferencias no se observan en el resto de las categorías de las estrategias de aprendizaje.

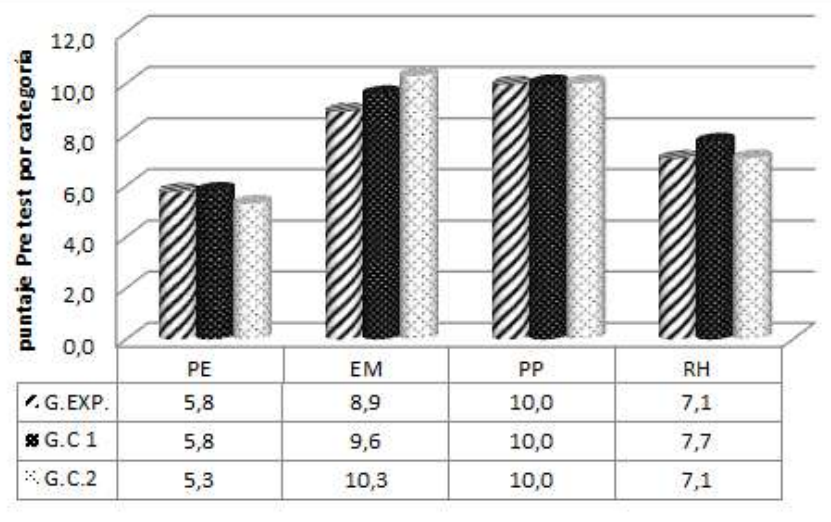

Fig. 6: Puntaje pre-test por categoría de estrategias de aprendizaje

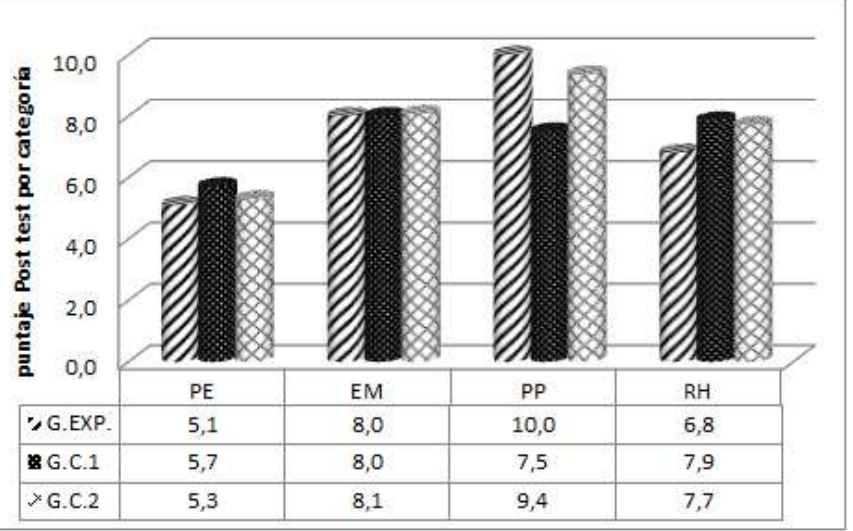

Fig. 7: Puntaje post-test por categoría de estrategias de aprendizaje

\section{Pre y post-test inventario de estrategias de aprendizaje en un mismo grupo en dos mediciones}

Para analizar los cambios en el procesamiento de la información en cada grupo, acorde a su orientación metodológica se aplica el cuestionario de estrategias de aprendizaje como pre y post-test, los resultados obtenidos se representan en la figura 8 que se realiza con el promedio de alumnos que en poseen puntuaciones entre los niveles normal y alto en cada una de las categorías del cuestionario, donde se muestra la evolución en cada grupo.

Los resultados correspondientes al grupo experimental, indican que existe un aumento en el número de alumnos que incrementa sus puntuaciones en la estrategia de Procesamiento Profundo (PP), sin embargo, no ocurre lo mismo en la estrategia de Procesamiento Elaborativo (PE). Mientas que el porcentaje de 
alumnos decrece en las estrategias superficiales de Estudio Metódico (EM) y Retención de Hecho (RH). A pesar de los cambios, no se encuentran diferencias significativas mediante la prueba de McNemar.

Los resultados de los grupos control 1 y 2, muestran un aumento en la estrategia Retención de Hecho (RH), contrario a lo que ocurre en las otras categorías del Inventario de R. Schmeck, demostrando que la forma en la que han trabajado ambos grupos control (resolución de guías de ejercicio y método peer), privilegia la mecanización de conceptos y procedimientos utilizados en física.

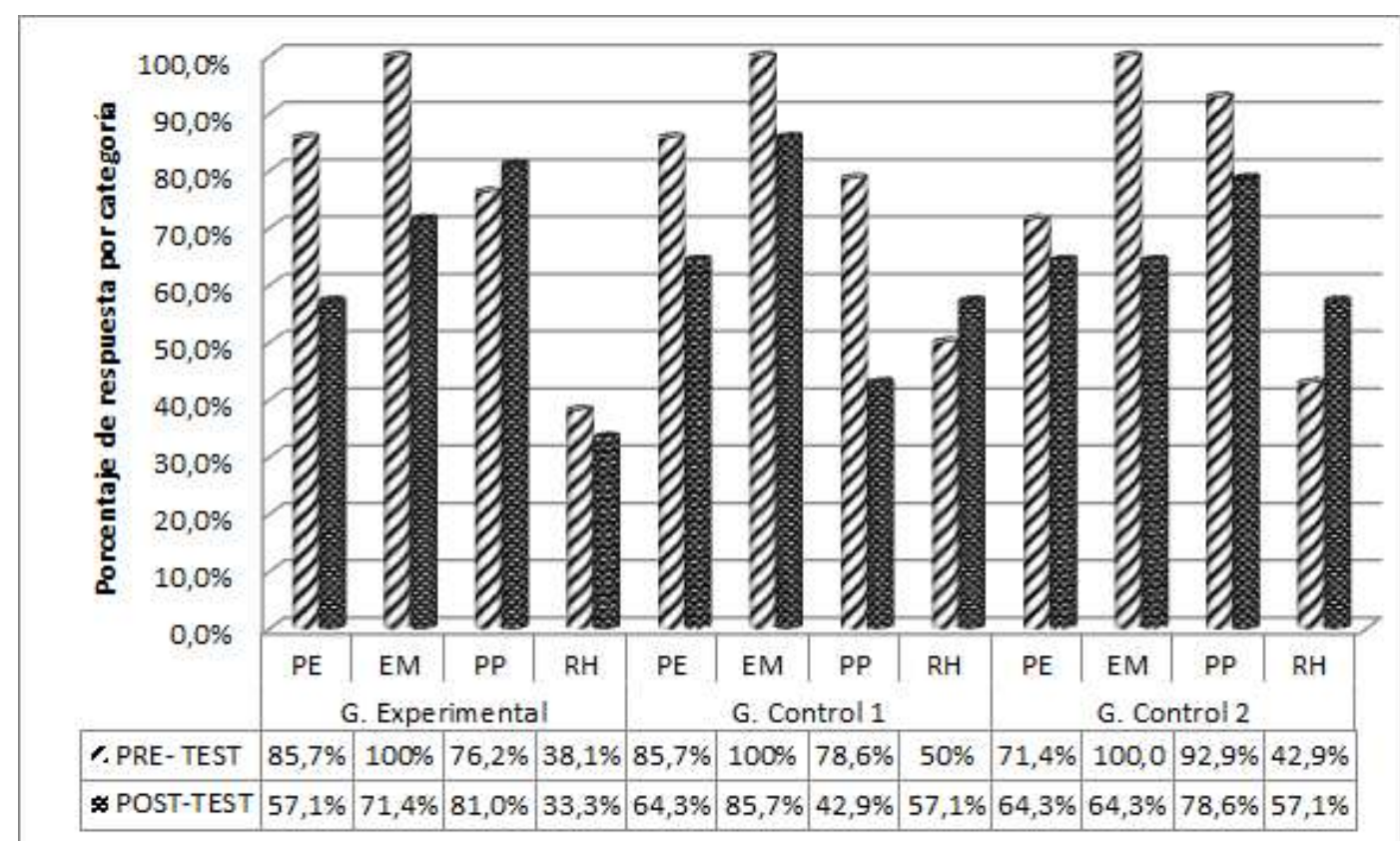

Fig. 8: Porcentaje de alumnos en nivel normal- alto en pre y post test versus estrategias de aprendizaje.

\section{RESULTADOS RENDIMIENTO ACADÉMICO}

A través de los instrumentos de evaluación se determinó el rendimiento académico de los alumnos intervenidos, los que se aplicaron a los tres grupos en forma simultánea, obteniendo las calificaciones y los promedios de cada grupo, lo que se muestra en la figura 9 para cada grupo.

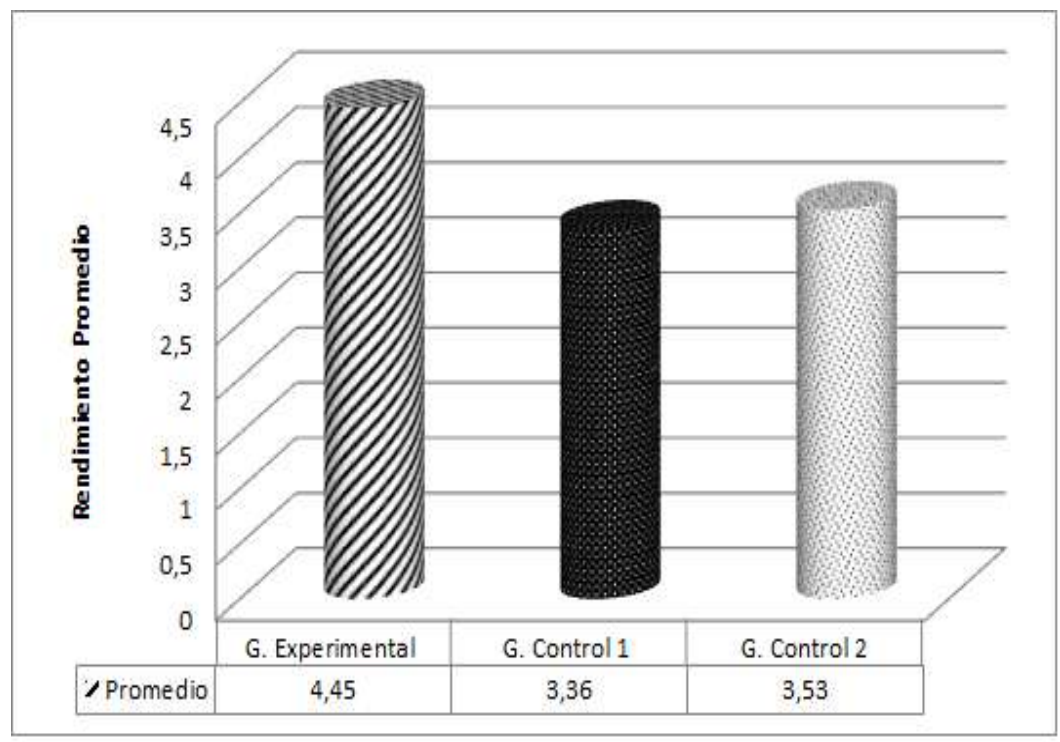

Fig. 9: Promedio de calificaciones obtenidas por los tres grupos.

Los resultados del gráfico muestran que el grupo experimental logra mejores calificaciones que los grupos control 1 y 2. De aquí se deduce que el programa de intervención propuesto favorece el éxito académico, se debe destacar que sólo el grupo experimental logra un promedio superior al mínimo de aprobación (4.0). La 
prueba de Kruskal-Wallis, establece un estadístico $H=14,80909$, y un nivel de significado $p=0,0006$ $(99,94 \%)$, que permite afirmar que existen diferencias reales y estadísticamente significativas en el rendimiento académico entre los grupos. La prueba de Tukey muestra que existen diferencias estadísticamente significativas que se decantan a favor del grupo experimental respecto al: a) Grupo Control $1 p=0,01122(98,9)$; b) G. Control 2, $p=0,007035$ (99,3); En cambio entre el Grupo Control 1 y Grupo Control 2, no existen diferencias significativas $p=0,838431(91,7 \%)$.

\section{DISCUSIÓN}

En función de los resultados es posible señalar que el programa favorece el desarrollo de ciertas capacidades creativas en los sujetos. Si bien los resultados del post-test no permiten establecer con claridad diferencias significativas a favor del grupo experimental, se observa una tendencia a la evolución en las dimensiones Artes y Escritura (AE) e Intereses Variados (IV) de los alumnos intervenidos con la propuesta didáctica.

Los grupos control 1 y 2 incrementan su puntuación en la estrategia Retención de Hecho $(R H)$, contrario a lo que ocurre en el grupo experimental, que ve disminuida su puntuación en 0,3 puntos, lo que implicaría que los mecanismos utilizados para la enseñanza y aprendizaje de la Física en los grupos control 1 y 2 , favorecen en los alumnos un aprendizaje superficial y reiterativo (aprendizaje mecánico) de los contenidos. En cambio, la diferenciación que logra el grupo experimental en el Procesamiento Profundo (PP) permite señalar que este grupo logra transitar desde un aprendizaje superficial y reiterativo, hacia un aprendizaje estratégico o profundo y elaborativo, que exigen la transferencia del contenido en situaciones nuevas, condición del aprendizaje significativo (Oses y Carrasco, 2013), lo que se evidencia en las puntuaciones altas en Estudio Metódico (EM) y procesamiento profundo PP. Si bien existe distanciamiento del aprendizaje mecánico, lo ideal hubiera sido que este movimiento se orientase hacia un aprendizaje con significado, sin embargo, esto no puede determinarse producto que no existen evidencias de cambios en la estrategia de Procesamiento Elaborativo, la otra condición del aprendizaje significativo.

La relación entre una renovación metodológica, el desarrollo de estrategias de aprendizaje y niveles de creatividad es un problema de investigación nuevo, es por ello que este trabajo es un aporte para futuros trabajos que buscan acumular evidencias en esta línea. Aun cuando los resultados muestran evidencias como para afirmar un impacto de la intervención en el desarrollo del procesamiento profundo una de las estrategias para alcanzar un aprendizaje significativo o estratégico, así como en el rendimiento académico, no es posible establecer juicios categóricos en relación a las demás variables. Ciertamente el desarrollo de estas estrategias cognitivas, procesamiento de la información y creatividad, exige tener presente: a) un mayor tiempo de intervención, lo que contribuiría a generar un mayor impacto en dichas variables, y b) una renovación metodológica en un mayor número de asignaturas simultáneas. En este contexto, se infiere que cualquier programa destinado a desarrollar las estrategias para aprender a aprender, debe considerar renovación metodológica y el tiempo de intervención.

\section{CONCLUSIONES}

El programa de intervención impacta positivamente en la estrategia procesamiento profundo (PP), relacionada con la identificación de las claves semánticas de la información, lo que se corrobora con la prueba estadística de Kruskal-Wallis, que arroja un estadístico $(H=6,677369) p=0,0355$, indicando un cambio estadísticamente significativo a favor del G. Experimental. Aún cuando una de las categorías del inventario de estrategias de aprendizaje fue modificada, no es posible determinar con certeza que los alumnos intervenidos hayan desarrollado las estrategias adecuadas para orientarse hacia un aprendizaje significativo (PE y PP), pues para ello debería haberse encontrado evidencia de un desarrollo similar en el procesamiento elaborativo (PE) en el G. experimental.

A través de la prueba de Kruskal-Wallis se determinó que la intervención metodológica a través del programa, logra generar diferencias significativas a un 99\%, $p=0,0006(H=14,80909)$ respecto al rendimiento académico (figura 9). Mediante de prueba de Tukey se observa que tales diferencias se producen a favor del grupo experimental. Por lo tanto, las actividades incluidas en el programa (ABP, pequeños experimentos, mapas conceptuales y $\mathrm{V}$ de Gowin) impactan positivamente en el rendimiento académico de los sujetos intervenidos.

\section{AGRADECIMIENTOS:}

La presente investigación recoge parte de los resultados obtenidos en el marco del proyecto Fondecyt №1120767; "Hacia un programa para desarrollar estrategias cognitivas desde la física". Financiado por el Fondo de Desarrollo Científico y Tecnológico de Chile. 


\section{REFERENCIAS}

Ausubel, D., J. Novak y H. Hanesian, Psicología educativa. Un punto de vista cognoscitivo. Ed. Trillas. México (1983).

Cano, F. y F. Justicia, Los estilos de aprendizaje en psicología y educación. En Psicología de la instrucción: componentes cognitivos y afectivos del aprendizaje escolar, 2, 87-110. Barcelona: EUB (1998).

Carbonero, M. Á., L.J. Martín-Antón, J.M. Román y N. Reoyo, Efecto de un programa de entrenamiento al profesorado en la motivación, clima del aula y estrategias de aprendizaje de su alumnado. Revista Iberoamericana de Psicología Y Salud, 1(2): 117-138. (2010)

Carpio, C., C. Canales, G. Morales, R. Arroyo y H. Silva, Inteligencia, creatividad y desarrollo psicológico. Acta Colombiana de Psicología, 10(2): 41-50 (2007).

Chrobak, R. Una enseñanza creativa, para obtener aprendizajes creativos. Cuadernos FHyCS-UNJu, 35: 115-129 (2008).

Cid, S., El uso de estrategias de aprendizaje y su correlación con la motivación de logro en los estudiantes. Revista Iberoamericana sobre Calidad, Eficacia y Cambio en Educación, 6(3): 101-120 (2008).

Cohen, L. y L. Manion, L., Métodos de investigación educativa. La muralla S. A, pp. 10-100. España (1990).

Donolo, D. y M.C. Rinaudo, Perspectivas y experiencias creativas para estudiantes universitarios. Cuadernos de FHyCS-UNJu, 35: 91-113 (2008).

Fernández, O.M., M. Martínez y R. Melipillán, Estrategias de Aprendizaje y Autoestima. Su relación con la ermanencia y deserción universitaria. Estudios Pedagógicos, XXXXV 1: 27-45 (2009).

Flores, J., M.C. Caballero y M.A. Moreira, El laboratorio en la enseñanza de las ciencias: una visión integral en este complejo ambiente de aprendizaje. Revista de Investigación, 68 (33) 75-111 (2009).

Gargallo, B., Estrategias de aprendizaje, rendimiento $y$ otras variables relevantes en estudiantes universitarios. Revista de psicología general y aplicada, 1-2: 109-130 (2006).

Gil, J. y F. Solano, El uso de la Vee epistemológica en la resolución de problemas en Física. XXXIII Reunión Bienal de Real Sociedad Española de Física (2011).

López S., E. Angela e I. Solano, Modelación computacional apoyada en el uso del diagrama V para el aprendizaje de conceptos de dinámica newtoniana. Revista Electrónica de Enseñanza de las Ciencias, 10 (1): 202-226 (2011).

López, M. O. Enseñanza creatividad. Cuadernos FHyCS-Universidad de Jujuy, 35: 31-40 (2008).

Martín, E., L. A. García, A. Torbay, y T. Rodríguez, Estrategias de aprendizaje y rendimiento académico en estudiantes universitarios. International Journal of Psychology and Psychological Therapy 8 (3) 401-412 (2008).

Martínez, A., Las estrategias de aprendizaje. Radiografías necesarias para su comprensión. Revista Electrónica Actualidades Investigativas en Educación, 9 (2) 1-26 (2009).

Moreira, M. A. Diagramas V y aprendizaje significativo. Revista Chilena de Educación Científica, vol. 6, N. 2 (2007).

Oses, S. y L. Carrasco. Módulos alternativos en la enseñanza de las ciencias: estrategia didáctica orientada al logro de aprendizajes significativos. Formación Universitaria, 6(3) 39-52 (2013)

Perales, F. J. La imagen en la enseñanza de las ciencias: algunos resultados de investigación en la Universidad de Granada, España. Formación Universitaria, 1(4) 13-22 (2008).

Pulgar, J. y I. Sánchez, Creativity and physics learning as product of the intervention with conceptual maps and Gowin's V diagrams. Creative Education, 4, 13-20 (2013).

Reyes, M. Las estrategias creativas como factor de cambio en la actitud del docente para la enseñanza de la matemática. Sapiens, 4 (2), (2003). 
Sánchez, I. Evaluación de una Renovación Metodológica para un Aprendizaje Significativo de la Física. Formación Universitaria, 5(5) 51-65, (2012)

Sánchez, I.R, L. Neriz y F.J. Ramis. Design and application of learning environments based on integrative problems. European Journal of Engineering Education. 33(4), 445-452 (2008).

Sánchez, I. R., M.A. Moreira y M. C. Caballero, Implementación de una propuesta de aprendizaje significativo de la cinemática a través de la resolución de problemas. Revista chilena de ingeniería, 17(1), 27-41 (2009).

Sánchez I.R., M.A. Moreira, y M.C. Caballero. "Implementación de una renovación metodológica para un aprendizaje significativo en Física I". Latin American Journal of Physics Education. 5(2), 475-484 (2011).

Schmeck, R. Individual differences and learning strategies in learning and study strategies. Issues in assessment, instruction and evaluation. Academy Press. New York (1988).

Truffello, I. y F. Pérez, Adaptación en Chile del "Inventory of Learning Processes" de R.Schmeck. Boletín de Investigación. Pontificia Universidad Católica de Chile, 15: 109-120 (1998).

Vega, F. E. Portillo, M. Cano y B. Navarrete. Experiencias de Aprendizaje en Ingeniería Química: diseño, montaje y puesta en marcha de una unidad de destilación a escala laboratorio mediante el aprendizaje basado en problemas. Formación. Universitaria. 7(1) 13-22 (2014). 